IJMMS 25:11 (2001) 703-708

PII. S0161171201005993

http://ijmms.hindawi.com

(c) Hindawi Publishing Corp.

\title{
POWER SUBGROUPS OF HECKE GROUPS $H(\sqrt{n})$
}

\author{
NIHAL YILMAZ and I. NACI CANGÜL
}

(Received 6 April 2000 and in revised form 17 October 2000)

\begin{abstract}
Results in discrete group theory are applied to some Hecke groups to determine
\end{abstract} the group theoretical structure of power subgroups.

2000 Mathematics Subject Classification. Primary 11F06, 20H05, 20H10.

1. Introduction. Hecke groups $H(\lambda)$ have been introduced by E. Hecke (see [2]). They are subgroups of $\operatorname{PSL}(2, \mathbb{R})$ generated by $R(z)=-1 / z$ and $T(z)=z+\lambda$. Hecke asked the question, "For what values of $\lambda$ these groups are discrete?" In answering this question he proved that

$$
F_{\lambda}=\left\{z \in U:|z|>1,|\operatorname{Re} z|<\frac{\lambda}{2}\right\}
$$

is a fundamental region for $H(\lambda)$ if and only if $\lambda \geq 2$ and real or $\lambda=\lambda_{q}=2 \cos (\pi / q)$, $q \in \mathbb{N}, q \geq 3$. Therefore, $H(\lambda)$ is discrete only for these values of $\lambda$. The most important and interesting Hecke group is the modular group $H\left(\lambda_{3}\right)=\operatorname{PSL}(2, \mathbb{Z})$. Next two interesting Hecke groups are obtained for $q=4$ and $q=6$. As $\lambda_{4}=\sqrt{2}$ and $\lambda_{6}=\sqrt{3}, H(\sqrt{2})$ and $H(\sqrt{3})$ denote the Hecke groups corresponding to $\lambda_{4}$ and $\lambda_{6}$, respectively. One of the main reasons for $H(\sqrt{2})$ and $H(\sqrt{3})$ to be two of the most important Hecke groups is that apart from modular group, they are the only Hecke groups $H\left(\lambda_{q}\right)$ whose elements can be completely described. Here we deal with the cases $H(\sqrt{n}), n$ square-free integer. $H(\sqrt{n})$ consists of the set of all matrices of the following types:

(i) $\left(\begin{array}{cc}a & b \sqrt{n} \\ c \sqrt{n} & d\end{array}\right) ; a, b, c, d \in \mathbb{Z}, a d-n b c=1$,

(ii) $\left(\begin{array}{cc}a \sqrt{n} & b \\ c & d \sqrt{n}\end{array}\right) ; a, b, c, d \in \mathbb{Z}, n a d-b c=1$.

Those of type (i) are called even while those of type (ii) are called odd. Even elements form a subgroup of index 2 called the even subgroup [1].

Let $S=R T$ so that $S(z)=-1 /(z+\lambda)$. In the cases $H(\sqrt{n}), n=2,3, S$ is an element of order $q=2 n$. Thus $R^{2}=S^{q}=I$ and $R S=T$ is parabolic. It is known that $H(\sqrt{n})$ is isomorphic to the free product $C_{2} * C_{q}$. Therefore $H(\sqrt{n})$ has the signature $(0 ; 2, q, \infty)$, [1]. In the case $n>3$ square-free integer, $S$ is an element of infinite order and $H(\sqrt{n})$ is isomorphic to the free product $C_{2} * \mathbb{Z}$, [6]. The signature of $H(\sqrt{n})$ is $(0 ; 2, \infty ; 1)$. That is, all the groups $H(\sqrt{n}), n$ square-free integer, are triangle groups containing a parabolic element. It is well known that a triangle group $(2, m, n)$ acts on the sphere, Euclidean plane or hyperbolic plane according to $1 / m+1 / n>1 / 2,1 / m+1 / n=1 / 2$, and $1 / m+1 / n<1 / 2$, respectively, [3]. 
The purpose of this paper is to determine the structure of the groups $H^{m}(\sqrt{n})$ of the Hecke groups $H(\sqrt{n}), n$ is a square-free integer. The groups $H^{m}(\sqrt{n})$ are defined to be the subgroups generated by the $m$ th powers of all the elements of $H(\sqrt{n})$, for some positive integer $m$. $H^{m}(\sqrt{n})$ is called the $m$ th power subgroup of $H(\sqrt{n})$. As fully invariant subgroups, they are normal in $H(\sqrt{n})$.

From the definition, one can easily deduce that

$$
H^{m}(\sqrt{n})>H^{m k}(\sqrt{n})
$$

and that

$$
\left(H^{m}(\sqrt{n})\right)^{k}>H^{m k}(\sqrt{n}) .
$$

Using (1.2), it is easy to deduce that

$$
H^{m}(\sqrt{n}) \cdot H^{k}(\sqrt{n})=H^{(m, k)}(\sqrt{n})
$$

Here $(m, k)$ denotes the greatest common divisor of $m$ and $k$.

2. Structure of power subgroups. We now discuss the group theoretical structure of these subgroups. First we have the following theorem.

THEOREM 2.1. (i) Let $n=2$ or 3 . The normal subgroup $H^{2}(\sqrt{n})$ is isomorphic to the free product of infinite cyclic group $\mathbb{Z}$ and two finite cyclic groups of order $n$. Also

$$
\begin{gathered}
H(\sqrt{n}) / H^{2}(\sqrt{n}) \cong C_{2} \times C_{2}, \\
H(\sqrt{n})=H^{2}(\sqrt{n}) \cup R H^{2}(\sqrt{n}) \cup S H^{2}(\sqrt{n}) \cup R S H^{2}(\sqrt{n}), \\
H^{2}(\sqrt{n})=\left\langle S^{2}\right\rangle *\left\langle R S^{2} R\right\rangle *\left\langle R S R S^{2 n-1}\right\rangle .
\end{gathered}
$$

The elements of $H^{2}(\sqrt{n})$ are characterized by the property that the sums of the exponents of $R$ and $S$ are both even.

(ii) Let $n>3$ square-free integer. The normal subgroup $H^{2}(\sqrt{n})$ is the free product of three infinite cyclic groups.

Also

$$
\begin{gathered}
H(\sqrt{n}) / H^{2}(\sqrt{n}) \cong C_{2} \times C_{2}, \\
H(\sqrt{n})=H^{2}(\sqrt{n}) \cup R H^{2}(\sqrt{n}) \cup S H^{2}(\sqrt{n}) \cup R S H^{2}(\sqrt{n}), \\
H^{2}(\sqrt{n})=\left\langle S^{2}\right\rangle *\left\langle R S^{2} R\right\rangle *\left\langle R S R S^{-1}\right\rangle .
\end{gathered}
$$

The elements of $H^{2}(\sqrt{n})$ can be characterized by the requirement that the sums of the exponents of $R$ and $S$ are both even.

Proof. We use the Reidemeister-Schreier process to find a presentation of $H^{2}(\sqrt{n})$, [5]. We add the relation $X^{2}=1$ to the presentation of $H(\sqrt{n})$. This gives a presentation of $H(\sqrt{n}) / H^{2}(\sqrt{n})$ the order of which is the index. We have

$$
H(\sqrt{n}) / H^{2}(\sqrt{n})=\left\langle R, S ; R^{2}=S^{2}=(R S)^{2}=1\right\rangle=C_{2} \times C_{2} .
$$


Thus $\left|H(\sqrt{n}): H^{2}(\sqrt{n})\right|=4$. Now we choose $\{I, R, S, R S\}$ as a Schreier transversal for $H^{2}(\sqrt{n})$. Then we can form all possible products

$$
\begin{gathered}
S_{I R}=I R R^{-1}=I, \quad S_{I S}=I S S^{-1}=I, \quad S_{R^{2}}=R R I=I, \\
S_{R S}=R S(R S)^{-1}=I, \quad S_{S R}=S R(R S)^{-1}=S R S^{-1} R, \\
S_{S^{2}}=S S I=S^{2}, \quad S_{R S R}=R S R(S)^{-1}=R S R S^{-1}, \quad S_{R S^{2}}=R S^{2} R .
\end{gathered}
$$

Since $\left(R S R S^{-1}\right)=S R S^{-1} R$, we get $x_{1}=S^{2}, x_{2}=R S^{2} R$, and $x_{3}=R S R S^{-1}$ as the generators of $H^{2}(\sqrt{n})$. Clearly the elements of $H^{2}(\sqrt{n})$ satisfy the requirements of the theorem, that is, the sums of the exponents of $R$ and $S$ are both even for each element. Note that we have $S^{-1}=S^{3}, S^{-1}=S^{5}$ for $n=2, n=3$, respectively. Using the Reidemeister rewriting process, we get the relations

$$
\begin{gathered}
\tau(I R R I)=\tau(R R)=S_{I R} \cdot S_{R^{2}}=I, \\
\tau(R R R R)=S_{I R} \cdot S_{R^{2}} \cdot S_{I R} \cdot S_{R^{2}}=I, \\
\tau\left(S R R S^{-1}\right)=S_{I S} \cdot S_{S R} \cdot S_{R S R} \cdot S_{I S}^{-1}=I S R S^{-1} R R S R S^{-1}=I, \\
\tau\left(R S R R S^{-1} R\right)=S_{I R} \cdot S_{R S} \cdot S_{R S R} \cdot S_{S R} \cdot S_{R S}^{-1} \cdot S_{R^{2}}=I I R S R S^{-1} S R S^{-1} R I I=I .
\end{gathered}
$$

Therefore there are no nontrivial relations and $H^{2}(\sqrt{n})$ is the free product of three infinite cyclic groups generated by $x_{1}, x_{2}$, and $x_{3}$. As each of $R, S$, and $T$ goes to elements of order 2 , they have the following permutation representations:

$$
R \longrightarrow\left(\begin{array}{ll}
1 & 2
\end{array}\right)\left(\begin{array}{ll}
3 & 4
\end{array}\right), \quad S \longrightarrow\left(\begin{array}{lll}
1 & 3
\end{array}\right)\left(\begin{array}{ll}
2 & 4
\end{array}\right), \quad T \longrightarrow\left(\begin{array}{lll}
1 & 4
\end{array}\right)\left(\begin{array}{ll}
2 & 3
\end{array}\right) .
$$

By the permutation method (see [4, 7]), the signature of $H^{2}(\sqrt{2})$ is $(g ; 2,2, \infty, \infty)=$ $\left(g ; 2^{(2)}, \infty(2)\right)$ and the signature of $H^{2}(\sqrt{3})$ is $\left(g ; 3^{(2)}, \infty(2)\right)$. Since the signature of all the Hecke groups $H(\sqrt{n}), n>3$ square-free integer, is $(0 ; 2, \infty ; 1)$, we find the signature of $H^{2}(\sqrt{n}), n>3$ square-free integer, as $\left(g ; \infty^{(2)} ; 2\right)$. Now by the Riemann-Hurwitz formula, we have $g=0$ in all cases. Hence $H^{2}(\sqrt{n}), n>3$ square-free integer, is isomorphic to the free product of three $\mathbb{Z}$ 's and $H^{2}(\sqrt{2})$ is isomorphic to the free product of $\mathbb{Z}$ and two finite cyclic groups of order 2 and $H^{2}(\sqrt{3})$ is isomorphic to the free product of $\mathbb{Z}$ and two finite cyclic groups of order 3 .

THEOREM 2.2. Let $m$ be a positive odd integer. Then $H^{m}(\sqrt{2})=H(\sqrt{2})$.

Proof. Teh proof is clear as the quotient is trivial.

TheOREM 2.3. Let $m$ be a positive integer such that $m \equiv 2 \bmod 4$. Then $H^{m}(\sqrt{2})$ is the free product of the infinite cyclic group $\mathbb{Z}$ and $m$ finite cyclic groups of order two.

Proof. It is easy to show that the quotient group is isomorphic to the dihedral group $D_{m}$ of order $2 m$. The permutation representations of $R, S$, and $T$ are

$$
\begin{aligned}
& R \longrightarrow(12)(34) \cdots(2 m-12 m), \\
& S \longrightarrow(23)(45) \cdots(2 m 1), \\
& T \rightarrow(135 \cdots 2 m-1)(2 m 2 m-2 \cdots 42)
\end{aligned}
$$


Then $H^{m}(\sqrt{2})$ has signature $\left(0 ; 2^{(m)}, \infty, \infty\right)$, that is, $H^{m}(\sqrt{2})$ is the free product given in the statement of the theorem. If we denote the normal subgroup by $W_{m}(\sqrt{2})$, we have $W_{m}(\sqrt{2}) \cong \mathbb{Z} * \underbrace{C_{2} * \cdots * C_{2}}_{m \text { times }}$.

We have already proved that

$$
H^{m}(\sqrt{2})= \begin{cases}H(\sqrt{2}) & \text { if } m \text { is odd } \\ W_{m}(\sqrt{2}) & \text { if } m \equiv 2 \bmod 4\end{cases}
$$

Because of this we are only left to consider the case where $m$ is a multiple of four. Now let $m=4 k, k \in \mathbb{N}$. Then in $H(\sqrt{2}) / H^{m}(\sqrt{2})$ we have the relations $r^{2}=s^{4}=1$, where $r$ and $s$ are the images of $R$ and $S$, respectively, under the homomorphism of $H(\sqrt{2})$ to $H(\sqrt{2}) / H^{m}(\sqrt{2})$. These relations imply that $H^{m}(\sqrt{2})$ is a free group.

THEOREM 2.4. The normal subgroup $H^{3}(\sqrt{3})$ is the free product of four cyclic groups of order 2. Also

$$
\begin{gathered}
H(\sqrt{3}) / H^{3}(\sqrt{3}) \cong C_{3}, \\
H(\sqrt{3})=H^{3}(\sqrt{3}) \cup S H^{3}(\sqrt{3}) \cup S^{2} H^{3}(\sqrt{3}), \\
H^{3}(\sqrt{3})=\langle R\rangle *\left\langle S^{3}\right\rangle *\left\langle S R S^{5}\right\rangle *\left\langle S^{2} R S^{4}\right\rangle .
\end{gathered}
$$

Proof. The proof is similar to that of Theorem 2.1.

The following results are easy to see.

THEOREM 2.5. Let $m \equiv \pm 1 \bmod 6$. Then $H^{m}(\sqrt{3})=H(\sqrt{3})$.

THEOREM 2.6. Let $m \equiv \pm 2 \bmod 6$. Then $H^{m}(\sqrt{3})=W_{m}(\sqrt{3})$.

TheOrem 2.7. Let $m \equiv 3 \bmod 6$. Then $H^{m}(\sqrt{3})=H^{3}(\sqrt{3})$.

Therefore the only case left is that when $m$ is divisible by 6 . A similar discussion will show that $H^{m}(\sqrt{3})$ is free in this case.

THEOREM 2.8. The normal subgroup $H^{3}(\sqrt{n}), n>3$ square-free integer, is the free product of three cyclic groups of order 2 and an infinite cyclic group. Also

$$
\begin{gathered}
H(\sqrt{n}) / H^{3}(\sqrt{n}) \cong C_{3}, \\
H(\sqrt{n})=H^{3}(\sqrt{n}) \cup S H^{3}(\sqrt{n}) \cup S^{2} H^{3}(\sqrt{n}), \\
H^{3}(\sqrt{n})=\langle R\rangle *\left\langle S^{3}\right\rangle *\left\langle S R S^{-1}\right\rangle *\left\langle S^{2} R S^{-2}\right\rangle .
\end{gathered}
$$

Proof. If we add the relation $X^{3}=1$ to the presentation of $H(\sqrt{n})$ we have

$$
H(\sqrt{n}) / H^{3}(\sqrt{n})=\left\langle R, S ; R^{2}=1, X^{3}=1\right\rangle=\left\langle S ; S^{3}=1\right\rangle \cong C_{3} .
$$

Thus $\left|H(\sqrt{n}): H^{3}(\sqrt{n})\right|=3$. Let $\left\{I, S, S^{2}\right\}$ be a Schreier transversal for $H^{3}(\sqrt{n})$. Then all the possible products are

$$
\begin{aligned}
& S_{I R}=I R I=R, \quad S_{I S}=I S S^{-1}=I, \quad S_{S R}=S R S^{-1}, \\
& S_{S^{2}}=S S S^{-2}=I, \quad S_{S^{2} R}=S^{2} R S^{-2}, \quad S_{S^{3}}=S^{3} I=S^{3} .
\end{aligned}
$$


Therefore, $H^{3}(\sqrt{n})$ is generated by $x_{1}=R, x_{2}=S^{3}, x_{3}=S R S^{-1}$, and $x_{4}=S^{2} R S^{-2}$. Using the Reidemeister rewriting process, we get the relations

$$
\begin{gathered}
\tau(I R R I)=\tau(R R)=S_{I R} \cdot S_{R^{2}}=R^{2}=I, \\
\tau\left(S R R S^{-1}\right)=S_{I S} \cdot S_{S R} \cdot S_{S R} \cdot S_{I S}^{-1}=I S R S^{-1} S R S^{-1} I=I, \\
\tau\left(S S R R S^{-1} S^{-1}\right)=S_{I S} \cdot S_{S^{2}} \cdot S_{S^{2} R} \cdot S_{S^{2} R} \cdot S_{S^{2}}^{-1} \cdot S_{I S}^{-1}=I I S^{2} R S^{-2} S^{2} R S^{-2} I I=I .
\end{gathered}
$$

The permutation representations of $R, S$, and $T$ are

$$
R \longrightarrow(1)(2)(3), \quad S \longrightarrow\left(\begin{array}{lll}
1 & 2 & 3
\end{array}\right), \quad T \longrightarrow\left(\begin{array}{lll}
1 & 2 & 3
\end{array}\right) .
$$

Then $H^{3}(\sqrt{n})$ has the signature $\left(0 ; 2^{(3)}, \infty ; 1\right)$, that is, $H^{3}(\sqrt{n})$ is the free product given in the statement of the theorem.

THEOREM 2.9. Let $m$ be a positive odd integer and $n>3$ is a square-free integer. Then

$$
H^{m}(\sqrt{n}) \cong \mathbb{Z} * \underbrace{C_{2} * \cdots * C_{2}}_{m \text { times }} .
$$

Proof. Since $H(\sqrt{n}) / H^{m}(\sqrt{n})=\left\langle S ; S^{m}=I\right\rangle \cong C_{m}$, the permutation representations of $R, S$, and $T$ are

$$
R \longrightarrow(1)(2) \cdots(m), \quad S \longrightarrow(12 \cdots m), \quad T \longrightarrow(12 \cdots m) .
$$

By the permutation method, we find the signature of $H^{m}(\sqrt{n})$ as $\left(0 ; 2^{(m)}, \infty ; 1\right)$. Therefore, $H^{m}(\sqrt{n})$ is isomorphic to the free product of $m$ cyclic groups of order 2 and an infinite cyclic group.

Let $m$ be a positive even integer and $n>3$ is a square-free integer. Then we have

$$
H(\sqrt{n}) / H^{m}(\sqrt{n})=\left\langle R, S ; R^{2}=S^{m}=(R S)^{m}=I\right\rangle,
$$

that is, the factor group is the group whose signature $(2, m, m)$. If $m=2$, we have already seen that $H^{2}(\sqrt{n}) \cong \mathbb{Z} * \mathbb{Z} * \mathbb{Z}$ which is a normal subgroup of genus 0 , then $H(\sqrt{n}) / H^{2}(\sqrt{n})$ is a group of automorphisms of a sphere with two boundary components and two punctures. If $m=4$, we have a normal subgroup acting on the Euclidean plane. Because, in this case the factor group $(2,4,4)$ is a group of infinite order and $1 / 4+1 / 4=1 / 2$. If $m \geq 6$ and even, the factor group $(2, m, m)$ is a group of infinite order and $1 / m+1 / m=2 / m<1 / 2$. Therefore, in this case we have a normal subgroup acting on the hyperbolic 2 -space (i.e., upper half plane).

ACKNOWLEDGEMENT. We would like to thank the referee for valuable comments and suggestions.

\section{REFERENCES}

[1] İ. N. Cangül and D. Singerman, Normal subgroups of Hecke groups and regular maps, Math. Proc. Cambridge Philos. Soc. 123 (1998), no. 1, 59-74. MR 98j:20071. Zbl 893.20036.

[2] E. Hecke, Ueber die Bestimmung Dirichletscher Reihen durch ihre Funktionalgleichung, Math. Ann. 112 (1936), 664-699 (German). Zbl 014.01601. 
[3] G. A. Jones, Graph imbeddings, groups, and Riemann surfaces, Algebraic Methods in Graph Theory, Vol. I, Conf. Szeged 1978 (Amsterdam), Colloq. Math. Soc. Janos Bolyai, vol. 25, North-Holland, 1981, pp. 297-311. MR 83b:05061. Zbl 473.05028.

[4] C. Maclachlan, Maximal normal Fuchsian groups, Illinois J. Math. 15 (1971), 104-113. Zbl 203.39201.

[5] W. Magnus, A. Karrass, and D. Solitar, Combinatorial Group Theory, 2nd revised ed., Interscience Publishers [John Wiley \& Sons ], New York, 1966, Presentations of groups in terms of generators and relations. MR 34\#7617. Zbl 138.25604.

[6] Yilmaz N. and İ. N. Cangül, On the group structure and parabolic points of Hecke group $H(\lambda)$, to appear.

[7] D. Singerman, Subgroups of Fuschian groups and finite permutation groups, Bull. London Math. Soc. 2 (1970), 319-323. MR 43\#7519. Zbl 206.30804.

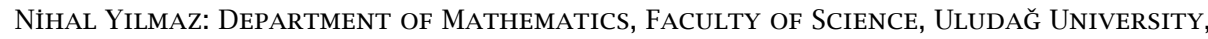
16059 BURSA, TURKEY

E-mail address: nyi1maz@u1udag.edu.tr

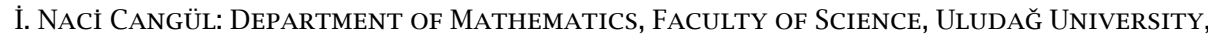
16059 BURSA, TURKEY

E-mail address: cangu1@u7udag.edu.tr 


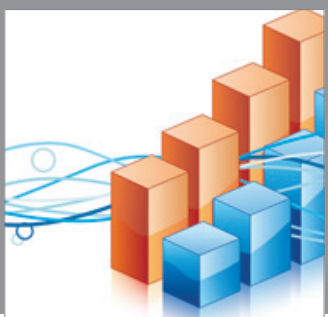

Advances in

Operations Research

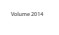

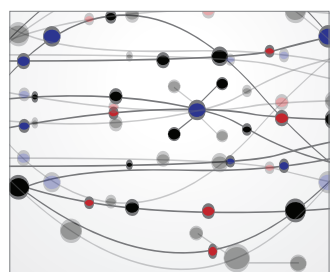

\section{The Scientific} World Journal
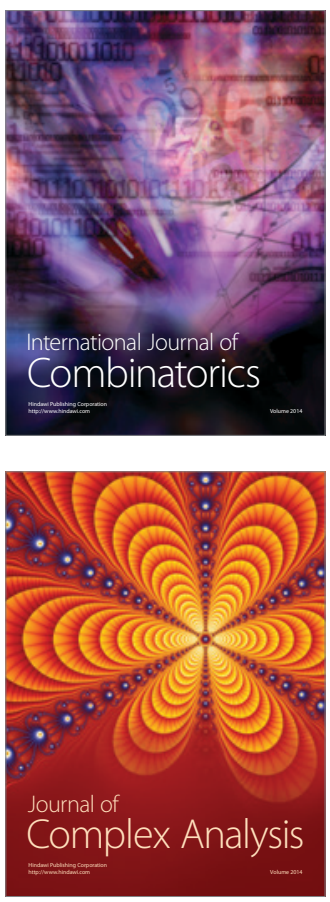

International Journal of

Mathematics and

Mathematical

Sciences
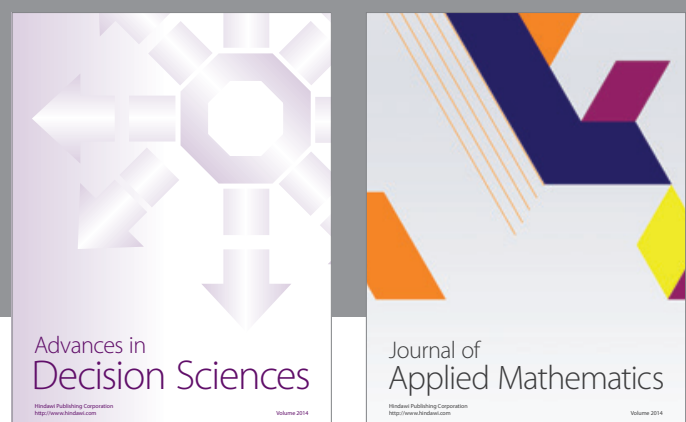

Journal of

Applied Mathematics
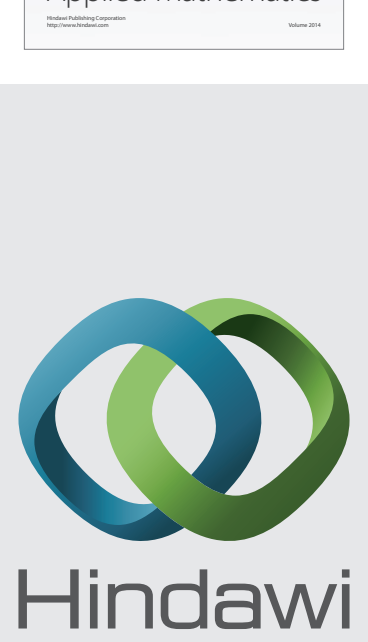

Submit your manuscripts at http://www.hindawi.com
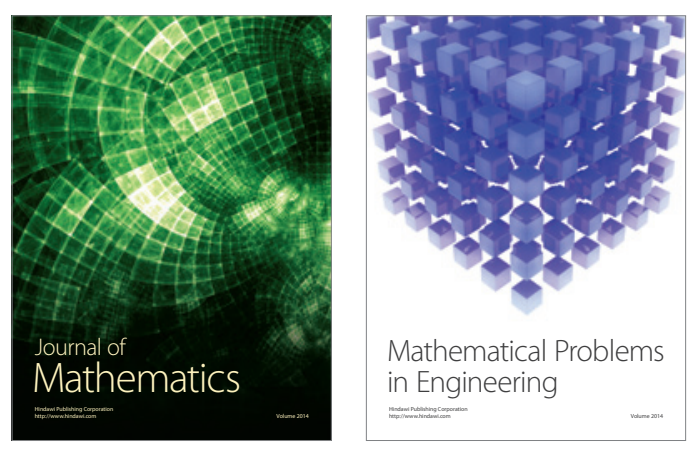

Mathematical Problems in Engineering
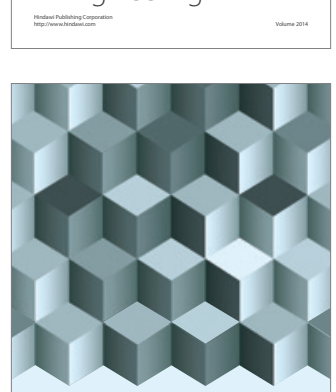

Journal of

Function Spaces
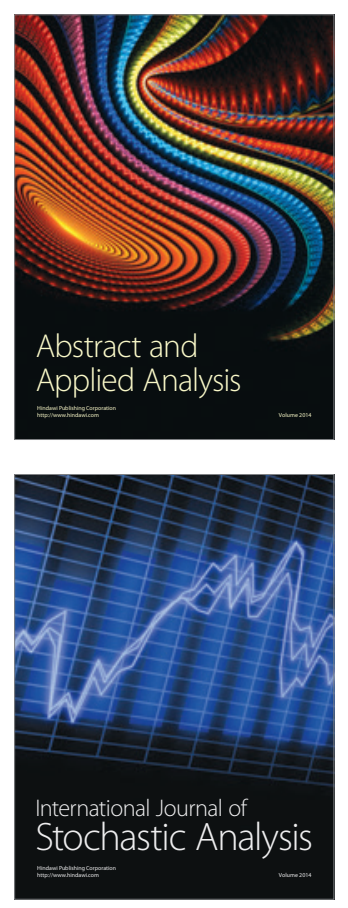

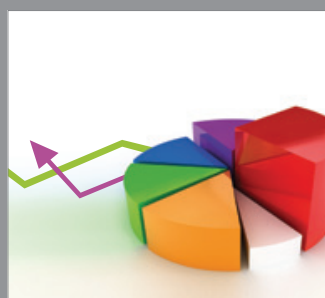

ournal of

Probability and Statistics

Promensencen
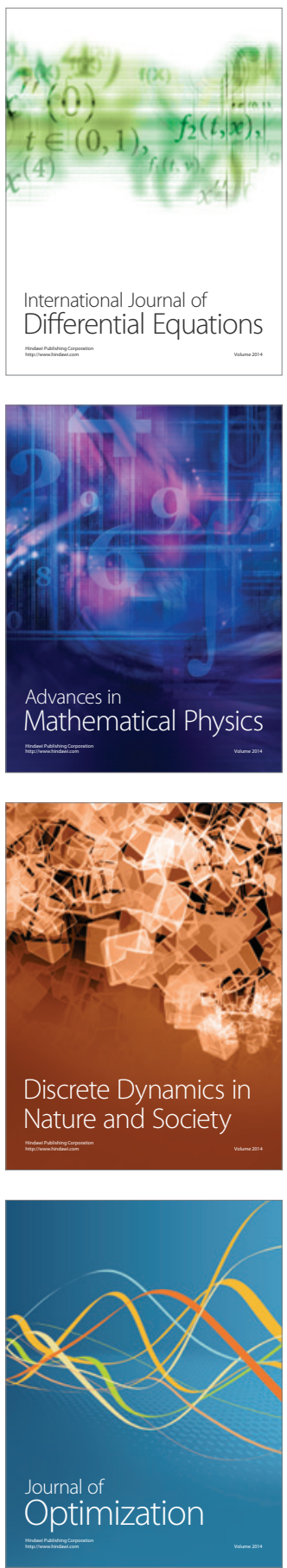